\title{
DYNAMICS IN THE CHARGED RESTRICTED CIRCULAR THREE-BODY PROBLEM
}

\author{
J.F. PALACIÁN, C. VIDAL, J. VIDARTE, AND P. YANGUAS
}

\begin{abstract}
The existence and stability of periodic solutions for different types of perturbations associated to the Charged Restricted Circular Three Body Problem (shortly, CHRCTBP) is tackled using reduction and averaging theories as well as the technique of continuation of Poincare for the study of symmetric periodic solutions. The determination of KAM 2-tori encasing some of the linearly stable periodic solutions is proved. Finally, we analyze the occurrence of Hamiltonian-Hopf bifurcations associated to some equilibrium points of the CHRCTBP.
\end{abstract}

\section{INTRODUCTION}

The charged three-body problem was introduced in [13] and has been studied by several authors[1, 2 ]. Some interesting results regarding this dynamical system from different points of view can be found, for example, in $[4,6,25,18,7,8,16,17]$.

We consider a planar charged restricted circular three-body problem (shortly, CHRCTBP) which was studied previously in [29]. Here, the two primaries with masses $1-\mu$ and $\mu(\mu \in(0,1 / 2))$ and electrostatic charges $q_{1}, q_{2} \in \mathbb{R}$, respectively, are moving in a circular orbit of the charged two-body problem while the infinitesimal particle is moving on the plane of the primaries attracted by Gravitational and Coulombian forces generated by them. According to [29] the Hamiltonian function associated to this problem in rotating coordinates $\left(x_{1}, x_{2}, y_{1}, y_{2}\right)$ is

$$
\mathcal{H}=\frac{1}{2}\left(y_{1}^{2}+y_{2}^{2}\right)-\left(x_{1} y_{2}-x_{2} y_{1}\right)-\frac{\beta_{1}^{3}(1-\mu)}{\sqrt{\left(x_{1}+\mu\right)^{2}+x_{2}^{2}}}-\frac{\beta_{2}^{3} \mu}{\sqrt{\left(x_{1}+\mu-1\right)^{2}+x_{2}^{2}}},
$$

with $\beta_{1}^{3}=1-\alpha q_{1} /(1-\mu), \beta_{2}^{3}=1-\alpha q_{2} / \mu$ satisfying $\left(1-\beta_{1}^{3}\right)\left(1-\beta_{2}^{3}\right)<\alpha^{2}, \alpha \in \mathbb{R}$. The Hamiltonian system associated to (1) is

$$
\begin{aligned}
\dot{x}_{1}=\frac{\partial \mathcal{H}}{\partial y_{1}}, & \dot{y}_{1}=-\frac{\partial \mathcal{H}}{\partial x_{1}}, \\
\dot{x}_{2}=\frac{\partial \mathcal{H}}{\partial y_{2}}, & \dot{y}_{2}=-\frac{\partial \mathcal{H}}{\partial x_{2}},
\end{aligned}
$$

which represents an autonomous system with two degrees of freedom depending on three parameters.

As it was discussed in [29] our model generalizes other restricted problems, namely, the classical Newtonian circular restricted three-body problem $\beta_{1}=\beta_{2}=1$ and the photogravitational threebody problem. Therefore this is one of the reasons of analyzing the dynamics of (2). A first step was initiated in [29], the existence of particular equilibrium points, namely, the collinear equilibrium points $L_{1}^{\text {coll }}, L_{2}^{\text {coll }}$ and $L_{3}^{\text {coll }}$ and the isosceles triangle equilibrium $L_{4}^{i s o}$ and $L_{5}^{\text {iso }}$ were characterized

1991 Mathematics Subject Classification. 34C15, 34C20, 34C25, 37J40, 70K65.

Key words and phrases. Charged restricted circular three body problem; averaging, normalisation and reduction; reduced space and invariants; Reeb's Theorem; periodic solutions and linear stability; KAM tori; bifurcations.

The authors are partially supported by Project MTM 2014-59433-C2-1-P of the Ministry of Economy and Competitiveness of Spain. 
in an explicit way as functions of the parameters $\mu$ and $\beta=\beta_{1}=\beta_{2}$. The linear and nonlinear stability of these equilibrium points was studied and the curves of resonance were analyzed in detail.

The purpose of this paper is to get a deeper insight in the CHRCTBP. More precisely, we search for different families of periodic solutions by combining symplectic reduction with averaging theories of Hamiltonian systems. Moreover we apply Poincaré's continuation method of symmetric periodic solutions by using the symmetry $\mathcal{S}_{1}:\left(x_{1}, x_{2}, y_{1}, y_{2}\right) \longrightarrow\left(x_{1},-x_{2},-y_{1}, y_{2}\right)$ of system (2). We also achieve the existence of KAM tori of dimension two close to some of the previous stable periodic solutions. Finally we establish the existence of Hamiltonian-Hopf bifurcations for the isosceles equilibrium points.

As it should be expected we recover the results existing in the literature for the Newtonian version of the planar restricted circular problem, because this case corresponds to $\beta_{1}=\beta_{2}=1$. However, for some of our achievements we need to perform the normalization of the Hamiltonian to higher orders since we have additional terms which are not present in the Newtonian case. Moreover, after checking the literature on the subject we emphasize that our result stated in Theorem 2.3 has not been considered even when the charges of the bodies are zero.

The main contribution of the paper is to provide various new results on the existence of periodic solutions and KAM 2-tori as well as the appearance of Hamiltonian-Hopf bifurcations in different regimes of the CHRCTBP. These achievements are scattered along the paper by means of the Theorems of Sections 2, 3, 4 and 5.

To carry out our outcomes we have organized the contents of the paper as follows.

In Section 2 we place the infinitesimal mass close to one of the primaries (lunar periodic orbits according to the notation in [20]) introducing a small parameter $\varepsilon$. Next, we normalize the problem in Delaunay coordinates and apply reduction theory (see for example, [24] and [28]) in order to characterize the possible points that generate periodic solutions. Then, we apply the averaging theory (Reeb's Theorem) in convenient coordinates of Poincaré-Delaunay type. Thereafter, we write the Hamiltonian in action-angle and apply Han-Li-Yi's Theorem [14] to prove the existence of two dimensional KAM tori, pointing out the excluded measure for the existence of quasi-periodic invariant tori. In Subsection 2.4, taking into account the symmetry $\mathcal{S}_{1}$, and writing Hamiltonian (1) in Poincaré-Delaunay coordinates, we are able to prove the existence of $\mathcal{S}_{1}$-symmetric solutions of fixed period close to any circular Kepler solutions.

In Section 3 we put the negligible mass far away from the two primaries (the so-called comet orbits according to the notation in [20]) introducing a small parameter $\varepsilon$. Next, we introduce the invariants related to the axial symmetry (see for example, [21] and [28]) as well as convenient symplectic coordinates on the reduced space so that we apply Reeb's Theorem followed by Neishtadt's result of Arnold's Theorem for proper degenerate Hamiltonians [3], to prove the existence of periodic solutions and KAM 2-tori, respectively. Also, we determine the measure of the set of tori disappearing under the perturbation.

The Poincaré charged problem is treated in Section 4 It consists in considering the mass parameter $\mu$ small, again using the ideas in [20]). Initially if the angular momentum $\gamma$ of a circular Keplerian solution is different from $\beta_{1}^{2}$ we can continue it to the full problem. Next, in Subsection 4.2 we prove the existence of $\mathcal{S}_{1}$-symmetric periodic solutions as continuation of elliptic and circular Kepler solutions where the period can be variable (for the elliptic solution) and fixed (for the circular one).

Section 5 is devoted to the occurrence of Hamiltonian-Hopf bifurcations related to the isosceles equilibrium points (i.e. $\beta_{1}=\beta_{2}$ and non-diagonalizable linearization around it). This analysis is carried out applying the results of bifurcation theory given in [20] and [15].

Finally, in Appendix 6 we recall Krein-Gel'fand's Theorem [27] for establishing strong stability of linear Hamiltonian systems. In addition we Reeb's Theorem [26] and Han-Li-Yi's Theorem [14], 
as well as the characterization of the Hamiltonian-Hopf bifurcation for Hamiltonian systems with two degrees of freedom.

\section{THE CHARGED LUNAR PROBLEM}

For us the charged lunar problem is the charged restricted circular three-body problem where the infinitesimal is close to one of the primaries. In order to introduce this class of problem, we first perform the linear change from $y_{2}$ and $x_{1}$ to $y_{2}-\mu$ and $x_{1}-\mu$, respectively, to bring one primary to the origin. Then, we introduce a small parameter $\varepsilon$ by replacing $y=\left(y_{1}, y_{2}\right)$ by $\varepsilon^{-1} \beta_{1}(1-\mu)^{1 / 3} y$ and $x=\left(x_{1}, x_{2}\right)$ by $\varepsilon^{2} \beta_{1}(1-\mu)^{1 / 3} x$. By doing so we restrict $\mathcal{H}$ to the case where the infinitesimal particle is moving around one of the primaries. This change is symplectic with multiplier $\varepsilon^{-1} \beta_{1}^{-2}(1-\mu)^{-2 / 3}$. The parameter $\varepsilon$ is a measure of the distance of the infinitesimal particle from the primary at the origin. Next we scale time by dividing $t$ by $\varepsilon^{3}$ and multiplying $\mathcal{H}$ by $\varepsilon^{3}$. Then we expand the resulting Hamiltonian in powers of $\varepsilon$ to get

$$
\mathcal{H}=\frac{1}{2}\left(y_{1}^{2}+y_{2}^{2}\right)-\frac{1}{\sqrt{x_{1}^{2}+x_{2}^{2}}}-\varepsilon^{3}\left(x_{1} y_{2}-x_{2} y_{1}\right)+\varepsilon^{4} \kappa x_{1}+\frac{1}{2} \varepsilon^{6} \nu\left(-2 x_{1}^{2}+x_{2}^{2}\right)+O\left(\varepsilon^{7}\right),
$$

where $\kappa=\left(1-\beta_{2}^{3}\right) \mu /\left(\beta_{1}(1-\mu)^{1 / 3}\right)$ and $\nu=\mu \beta_{2}^{3}$. The zeroth-order term, that we denote by $\mathcal{H}_{0}$, is the Hamiltonian of the planar Kepler problem and the $O\left(\varepsilon^{3}\right)$ term is the Coriolis force due to the use of a rotating frame.

2.1. Normalisation and reduction. We introduce mixed polar and Delaunay coordinates as in [28] so that the Hamiltonian is ready for the elimination of the mean anomaly $\ell$ to high order by means of a special Lie transformation well suited for perturbed Kepler problems, the so-called normalization of Delaunay [11, 12]. Hamiltonian (3) in these coordinates reads as

$$
\mathcal{H}_{\varepsilon}=-\frac{1}{2 L^{2}}-\varepsilon^{3} G+\varepsilon^{4} \kappa r \cos \theta-\frac{1}{4} \varepsilon^{6} \mu \nu r^{2}(1+3 \cos (2 \theta))+O\left(\varepsilon^{7}\right),
$$

where $(\ell, g, L, G)$ correspond to Delaunay elements: $\ell$ the mean anomaly, $g$ the argument of the pericenter, and $L$ the square of the semimajor axis. Since $G$ is the third component of the angular moment vector $\mathbf{G}=(0,0, G)$, it follows that $0 \leq|G| \leq L$ and $G$ can be positive, negative, or zero. Finally, $(r, \theta)$ are the usual polar coordinates.

Next, we normalize Hamiltonian (4) up to order six in the small parameter applying the Delaunay normalization. We arrive at

(5) $\mathcal{H}_{\varepsilon}=-\frac{1}{2 L^{2}}-\varepsilon^{3} G-\frac{3}{2} \varepsilon^{4} \kappa L \sqrt{L^{2}-G^{2}} \cos g-\frac{1}{8} \varepsilon^{6} \nu\left(5 L^{2}-3 G^{2}+15\left(L^{2}-G^{2}\right) \cos (2 g)\right)+O\left(\varepsilon^{7}\right)$.

This normalization is effectively the average of the perturbations over the periodic solutions of the planar Kepler problem, whose Hamiltonian in these coordinates is given by $\mathcal{H}_{0}=-\frac{1}{2 L^{2}}$.

Now, considering the integral manifold $\mathcal{N}_{0}(h)=\mathcal{H}_{0}^{-1}(h)$ with $h<0$, the reduced space of the planar Kepler problem, that we denote by $\mathcal{B}(h)$, is diffeomorphic to a 2 -sphere $S^{2}$ (see details in [24]), which is a symplectic compact manifold. This space is described by three invariants $\mathbf{a}=\left(a_{1}, a_{2}, a_{3}\right)$ defined by the combination $\mathbf{a}=\mathbf{G}+L \mathbf{A}$, where $\mathbf{A}$ is the Laplace-Runge-Lenz vector.

Explicitly they are

$$
a_{1}=e L \cos g, \quad a_{2}=e L \sin g, \quad a_{3}=G,
$$

where $e=\sqrt{1-G^{2} / L^{2}}$ represents the eccentricity. The nonzero Poisson brackets between the invariants are

$$
\left\{a_{1}, a_{2}\right\}=a_{3}, \quad\left\{a_{2}, a_{3}\right\}=a_{1}, \quad\left\{a_{3}, a_{1}\right\}=a_{2}
$$


Clearly, $\|\mathbf{a}\|=L$ and the vector a uniquely determines a bounded orbit of the Kepler problem on the energy level $\mathcal{N}_{0}(h)$. Points $(0,0, \pm L)$ correspond to the circular orbits, the circle $a_{3}=0$ (the equator) accounts for the collision orbits, and the other points on the sphere refer to elliptic motions. Hamiltonian (5) in terms of the invariants reads as

$$
\mathcal{H}_{\varepsilon}=-\frac{1}{2 L^{2}}-\varepsilon^{3} a_{3}-\frac{3}{2} \varepsilon^{4} \kappa L a_{1}-\frac{1}{8} \varepsilon^{6} \nu L^{2}\left(5 L^{2}+15 a_{1}^{2}-15 a_{2}^{2}-3 a_{3}^{2}\right)+O\left(\varepsilon^{7}\right) .
$$

The reduced space $\mathcal{B}(h)$, that is $S^{2}$, is given by the relations (6) and $a_{1}^{2}+a_{2}^{2}+a_{3}^{2}=L^{2}$. The reduced Hamiltonian is obtained from (8) truncating terms of order $\varepsilon^{4}$ and higher, dropping the constant terms and dividing the resulting Hamiltonian by $\varepsilon^{3}$, that is,

$$
\overline{\mathcal{H}}=-a_{3} .
$$

To obtain the vector field associated to (9) in the coordinates $\left(a_{1}, a_{2}, a_{3}\right)$ we use $(7)$ and $\dot{a}_{j}=$ $\sum_{k=1}^{3}\left\{a_{j}, a_{k}\right\} \partial \overline{\mathcal{H}} / \partial a_{k}$. So the equations of motion become

$$
\dot{a}_{1}=a_{2}, \quad \dot{a}_{2}=-a_{1}, \quad \dot{a}_{3}=0 .
$$

Proposition 2.1. On the reduced space $\mathcal{B}(h)$, system (10) has two critical points of circular type given by $(0,0, L)$ and $(0,0,-L)$.

Proof. System (10) has to be solved taking into account the restrictions of (6) and $a_{1}^{2}+a_{2}^{2}+a_{3}^{2}=$ $L^{2}$.

2.2. Symplectic coordinates on the reduced space $\mathcal{B}(h)$. We consider now the symplectic coordinates defined in the neighborhoods of the critical points of Proposition 2.1. Specifically, we make use of the symplectic coordinates $(Q, P)$ defined through the transformations $T^{ \pm}: \Omega^{ \pm} \rightarrow \mathbb{R}^{2}$ given by

$$
Q=\sqrt{2} \sqrt{L \mp G} \cos g, \quad P= \pm \sqrt{2} \sqrt{L \mp G} \sin g,
$$

where $\Omega^{ \pm}=\{(G, L): \pm G \leq L\}$. The upper sign applies for $(0,0, L)$ whereas the lower one is used for $(0,0,-L)$. Combining the relations given in (6) and (11), one may put the coordinates $Q$ and $P$ in terms of the a. After simplifying the resulting expressions one gets

$$
Q=\frac{\sqrt{2} a_{1}}{\sqrt{L \pm a_{3}}}, \quad P= \pm \frac{\sqrt{2} a_{2}}{\sqrt{L \pm a_{3}}} .
$$

Thence that the maps $\psi_{1}, \psi_{2}: U_{j} \rightarrow \mathbb{R}^{2}, \psi_{j}(\mathbf{a})=(Q, P)$ defined by the equations (12), are local charts for the reduced space $\mathcal{B}(h)$, where $U_{j}=\left\{\mathbf{a}=\left(a_{1}, a_{2}, a_{3}\right) \in \mathcal{B}(h):(-1)^{j+1} a_{3}>0\right\}$ for $j=1,2$. Next, the critical points of Proposition 2.1 are identified with the origin of $\mathbb{R}^{2}$ by means of the atlas $\mathcal{A}=\left\{\psi_{1}, \psi_{2}\right\}$.

In order to introduce the coordinates $(Q, P)$ in Hamiltonians (5) and (9), first note that

$$
\frac{1}{2}\left(Q^{2}+P^{2}\right)=L \mp G=L \mp a_{3},
$$

and also

$$
a_{1}^{2}=\frac{Q^{2}}{2}\left(L \pm a_{3}\right), \quad a_{2}^{2}=\frac{P^{2}}{2}\left(L \pm a_{3}\right) .
$$

Thus, in these coordinates Hamiltonian (5) takes the form

$$
\begin{aligned}
\mathcal{H}_{\varepsilon}= & -\frac{1}{2 L^{2}} \pm \varepsilon^{3}\left(\frac{1}{2}\left(Q^{2}+P^{2}\right)-L\right)-\frac{3}{4} \varepsilon^{4} \kappa L \sqrt{4 L-Q^{2}-P^{2}} \\
& -\frac{1}{16} \varepsilon^{6} \nu L^{2}\left(4 L^{2}+36 L Q^{2}-24 L P^{2}-9 Q^{4}-3 Q^{2} P^{2}+6 P^{4}\right)+O\left(\varepsilon^{7}\right) .
\end{aligned}
$$


Now, to obtain the Hamiltonian on the reduced space $\mathcal{B}(h)$, we replace $L$ by $1 / \sqrt{-2 h}$ in $(9)$, remove constant terms and rescale time by dividing by $\varepsilon^{3}$, ending up with

$$
\begin{aligned}
\overline{\mathcal{H}}_{\varepsilon}= & \pm \frac{1}{2}\left(Q^{2}+P^{2}\right)-\frac{3}{4} \varepsilon \kappa L Q \sqrt{4 L-Q^{2}-P^{2}} \\
& -\frac{3}{16} \varepsilon^{3} \nu L^{3}\left(2 P^{2}-3 Q^{2}\right)\left(Q^{2}+P^{2}-4 L\right)+O\left(\varepsilon^{4}\right) .
\end{aligned}
$$

In this way, the truncated reduced Hamiltonian is given by

$$
\overline{\mathcal{H}}= \pm \frac{1}{2}\left(Q^{2}+P^{2}\right)
$$

2.3. Periodic solutions and KAM tori. In what follows we denote by

$$
p(t, \varepsilon)=\left(x_{1}(t, \varepsilon), x_{2}(t, \varepsilon), y_{1}(t, \varepsilon), y_{2}(t, \varepsilon)\right)
$$

a solution of system $(3)$ and by $p^{*} \in \mathcal{N}_{0}(h)$ the periodic solution associated to the critical point $\bar{p} \in S^{2}$.

Theorem 2.1. The charged lunar problem has two families of near-circular periodic solutions linearly stable with characteristic multipliers $1,1,1 \pm 2 \varepsilon^{3} T+O\left(\varepsilon^{4}\right)$. The radii and periods of these solutions are very small, $\|x\| \approx \varepsilon^{2} L^{2}$ and $T(\varepsilon) \approx 2 \pi \varepsilon$.

Proof. Clearly, the origin is a critical point of (15) and the Hessian matrix $D^{2} \overline{\mathcal{H}}$ at the origin is non-degenerate. Thus, by Reeb's Theorem 6.2 the proof of the theorem follows. Also, since the matrix $A=\mathbb{J} D^{2} \overline{\mathcal{H}}$ (J stands for the usual skew-symplectic matrix of mechanics) is strongly stable then the periodic solutions are linearly stable.

Theorem 2.2. The near-circular periodic solutions of the charged lunar problem are enclosed by invariant KAM 2-tori for small enough $\varepsilon$. The excluded measure for the existence of quasi-periodic invariant tori is of order $O\left(\varepsilon^{3}\right)$.

Proof. First, we introduce action-angle variables defined through the relations

$$
Q=\sqrt{2 I} \cos \theta, \quad P=\sqrt{2 I} \sin \theta .
$$

Thence the Hamiltonian (14) assumes the form

$$
\overline{\mathcal{H}}_{\varepsilon}= \pm I-\frac{3}{2} \varepsilon \kappa L \sqrt{I} \sqrt{2 L-I} \cos \theta-\frac{3}{4} \varepsilon^{3} \nu L^{2} I(2 L-I)\left(-2+5 \cos ^{2} \theta\right)+O\left(\varepsilon^{4}\right),
$$

then average over $\theta$ to get

$$
\overline{\mathcal{H}}= \pm I+\frac{9}{8} \varepsilon^{2} \kappa^{2} L^{2}(I-L)+O\left(\varepsilon^{3}\right) .
$$

In order to apply Han-Li-Yi's Theorem [14], we incorporate to (18) the terms associated to the action $L$ dropped in the process of normalization and undo the time scalings, so we get Hamiltonian (3) in the local coordinates that have been introduced through the process. Specifically we arrive at

$$
\mathcal{H}_{\varepsilon}=h_{0}(L)+\varepsilon^{3} h_{1}(L, I)+\varepsilon^{5} h_{2}(L, I)+O\left(\varepsilon^{6}\right),
$$

where

$$
h_{0}=-\frac{1}{2 L^{2}}, \quad h_{1}= \pm(I-L), \quad h_{2}=\frac{9}{8} \kappa^{2} L^{2}(I-L) .
$$

Now, we are in position to apply Han-Li-Yi's Theorem 6.4 taking $n=2, a=1, m_{1}=3, n_{0}=1$, $n_{1}=2, I^{n_{0}}=\bar{I}^{n_{0}}=L, I^{n_{1}}=(L, I)$, and $\bar{I}^{n_{1}}=I$. In this case, the vector of frequencies has dimension 2 and is given by

$$
\Omega(L, I)=\left(L^{-3}, \pm 1\right)
$$


The $2 \times 2$ matrix whose columns are $\Omega(I), \partial \Omega / \partial L$ and $\partial \Omega / \partial I$ reads as

$$
M_{\Omega}=\left[\begin{array}{ccc}
L^{-3} & -3 L^{-4} & 0 \\
\pm 1 & 0 & 0
\end{array}\right]
$$

and has rank two. Therefore, Han-Li-Yi's Theorem guarantees the existence of KAM 2-tori enclosed for $\varepsilon$ small the near-circular periodic solutions. Setting $b=\sum_{j=1}^{a} m_{j}\left(n_{j}-n_{j-1}\right)$, we obtain $b=3$ and according to Theorem 6.4 we have $s=1$, therefore the excluded measure for the existence of quasi-periodic invariant tori is of order $O\left(\varepsilon^{3}\right)$.

2.4. Symmetric periodic solutions. In the charged restricted lunar problem also we can find $\mathcal{S}_{1}$-symmetric periodic solutions with fixed period near to circular solutions of the Kepler problem.

Lemma 2.1. A solution of the charged restricted circular three-body problem (1) that crosses the line of syzygy orthogonally at a time $t=0$ and later at a time $t=T / 2>0$ is T-periodic and symmetric with respect to the line of syzygy.

See the proof in [20].

Let us introduce Poincaré-Delaunay coordinates, $\left(Q_{1}, Q_{2}, P_{1}, P_{2}\right)$, by

$$
\begin{array}{ll}
Q_{1}=\ell+g, & Q_{2}=\sqrt{2(L-G)} \cos \ell, \\
P_{1}=L, & P_{2}=\sqrt{2(L-G)} \sin \ell .
\end{array}
$$

An orthogonal crossing on the line of syzygy at a time $t_{0}$ is

$$
Q_{1}\left(t_{0}\right)=\pi n, \quad Q_{2}\left(t_{0}\right)=0, \quad n \in \mathbb{Z} .
$$

In these coordinates the Hamiltonian (3) is written as

$$
\mathcal{H}=-\frac{1}{2 P_{1}^{2}}+\varepsilon^{3}\left(-P_{1}+\frac{1}{2}\left(Q_{2}^{2}+P_{2}^{2}\right)\right)+O\left(\varepsilon^{4}\right),
$$

and the vector field of $(21)$ is

$$
\begin{array}{ll}
\dot{Q}_{1}=1 / P_{1}^{3}-\varepsilon^{3}+O\left(\varepsilon^{4}\right), & \dot{P}_{1}=O\left(\varepsilon^{4}\right), \\
\dot{Q}_{2}=\varepsilon^{3} P_{2}+O\left(\varepsilon^{4}\right), & \dot{P}_{2}=-\varepsilon^{3} Q_{2}+O\left(\varepsilon^{4}\right) .
\end{array}
$$

Now, we denote by $\psi\left(t, \mathbf{Y}_{0}, \varepsilon\right)=\left(Q_{1}(t, \varepsilon), Q_{2}(t, \varepsilon), P_{1}(t, \varepsilon), P_{2}(t, \varepsilon)\right)$ a solution of system (3) in Poincaré-Delaunay coordinates with initial condition $\mathbf{Y}_{0}=\left(\pi n, 0, p_{1}, p_{2}\right)$. Also, with respect to this initial condition we introduce the notation $\mathbf{Y}_{\varepsilon}=\left(\pi n, 0, p_{1}+O(\varepsilon), p_{2}+O(\varepsilon)\right)$.

Theorem 2.3. Let $m \in \mathbb{Z}, h \in \mathbb{R}^{-}$, and $T / 2=\pi m /(-2 h)^{3 / 2}$. Then the circular $T$-periodic solution of the Kepler problem with initial condition $\mathbf{Y}_{0}=(\pi n, 0,1 / \sqrt{-2 h}, 0)$ can be continued into the restricted charged lunar problem for $\varepsilon$ small. More precisely, for $\varepsilon$ sufficiently small there is an initial condition $\mathbf{Y}_{\varepsilon}$ such that $\psi\left(t, \mathbf{Y}_{\varepsilon}, \varepsilon\right)$ is a $\mathcal{S}_{1}$-symmetric solution with fixed period $T$.

Proof. Let $\psi(t, \mathbf{Y}, \varepsilon)$ be a circular solution of system $(22)$ with initial condition $\mathbf{Y}=\left(\pi n, 0, P_{1}, P_{2}\right)$ in a neighborhood of $\mathbf{Y}_{0}$. Solving system (22) we obtain

$$
Q_{1}(t, \mathbf{Y}, \varepsilon)=\pi n+P_{1}^{-3} t-\varepsilon^{3} t+O\left(\varepsilon^{4}\right), \quad Q_{2}(t, \mathbf{Y}, \varepsilon)=\varepsilon^{3} P_{2} t+O\left(\varepsilon^{4}\right) .
$$

Now, considering the functions

$$
f_{1}(t, \mathbf{Y}, \varepsilon)=\pi n+P_{1}^{-3} t-\varepsilon^{3} t+O\left(\varepsilon^{4}\right), \quad f_{2}(t, \mathbf{Y}, \varepsilon)=P_{2} t+O(\varepsilon)
$$

it is verified that

$$
Q_{1}\left(T / 2, \mathbf{Y}_{0}, 0\right)=f_{1}\left(T / 2, \mathbf{Y}_{0}, 0\right)=\pi(n+m), \quad Q_{2}\left(T / 2, \mathbf{Y}_{0}, 0\right)=f_{2}\left(T / 2, \mathbf{Y}_{0}, 0\right)=0
$$


Moreover, the solution has another orthogonal crossing at time $T / 2=\pi m /(-2 h)^{3 / 2}$ for $\varepsilon=0$, and so is $S_{1}$-symmetric. On the other hand, the determinant

$$
\left.\frac{\partial\left(f_{1}, f_{2}\right)}{\partial\left(P_{1}, P_{2}\right)}\right|_{\left(P_{1}=1 / \sqrt{-2 h}, P_{2}=0\right)}=\left|\begin{array}{cc}
-3(-2 h)^{2} T / 2 & 0 \\
0 & T / 2
\end{array}\right|=-3 \sqrt{-2 h} \pi m
$$

is nonzero. Thus, by the implicit function theorem there are functions $P_{1}(\varepsilon)=O(\varepsilon)$ and $P_{2}(\varepsilon)=$ $O(\varepsilon)$ such that

$$
Q_{1}\left(T / 2, \mathbf{Y}_{\varepsilon}, \varepsilon\right)=f_{1}\left(T / 2, \mathbf{Y}_{\varepsilon}, \varepsilon\right)=\pi(n+m) \quad \text { and } \quad Q_{2}\left(T / 2, \mathbf{Y}_{\varepsilon}, \varepsilon\right)=\varepsilon^{3} f_{2}\left(T / 2, \mathbf{Y}_{\varepsilon}, \varepsilon\right)=0
$$

for $\mathbf{Y}_{\varepsilon}=\left(\pi n, 0,1 / \sqrt{-2 h}+P_{1}(\varepsilon), P_{2}(\varepsilon)\right)$. In consequence, $\mathbf{Y}_{\varepsilon}$ is the initial condition for the $\mathcal{S}_{1^{-}}$ symmetric solution with fixed period $T$ asserted in the Theorem.

Remark 1. The integer $m$ is the number of revolutions that the particle describes around the solution circular.

\section{The ChARged COMET PROBlem}

Another way to introduce a small parameter is to consider orbits that are close to infinity. For us the charged comet problem is the restricted circular three-body problem where the infinitesimal is far from the primaries. In order to study this problem, we introduce a small parameter $\varepsilon$ in the Hamiltonian (1), through the scaling of variables $x \rightarrow \varepsilon^{-2} x, y \rightarrow \varepsilon y$. This change is symplectic with multiplier $\varepsilon$. The Hamiltonian becomes

$$
\mathcal{H}_{\varepsilon}=-\left(x_{1} y_{2}-x_{2} y_{1}\right)+\varepsilon^{3}\left(\frac{1}{2}\left(y_{1}^{2}+y_{2}^{2}\right)-\frac{1}{\sqrt{x_{1}^{2}+x_{2}^{2}}}\right)+O\left(\varepsilon^{6}\right) .
$$

Now $\varepsilon$ small means that the infinitesimal is near infinity, and (24) says that near infinity the Coriolis force $\mathcal{G}=x_{2} y_{1}-x_{1} y_{2}$ dominates, and the next most important force looks like a Kepler problem with both primaries at the origin.

3.1. Reduction. First, we introduce the invariants by the axial symmetry associated to Hamiltonian $\mathcal{G}$. These invariants that we will denote by $b_{1}, b_{2}, b_{3}$ and $b_{4}$ (see details in [21]) are just

$$
b_{1}=x_{1}^{2}+x_{2}^{2}, \quad b_{2}=y_{1}^{2}+y_{2}^{2}, \quad b_{3}=x_{1} y_{2}+x_{2} y_{2}, \quad b_{4}=x_{2} y_{1}-x_{1} y_{2} .
$$

with the constraint

$$
b_{1} b_{2}=b_{3}^{2}+b_{4}^{2}, \quad b_{1}, b_{2} \geq 0 .
$$

The nonzero Poisson brackets are

$$
\left\{b_{1}, b_{2}\right\}=4 b_{3}, \quad\left\{b_{3}, b_{2}\right\}=2 b_{2}, \quad\left\{b_{3}, b_{1}\right\}=-2 b_{1} .
$$

After fixing $b_{4}=\gamma($ or $\mathcal{G}=-\gamma)$, the integral manifold $\mathcal{N}_{0}(\gamma)=\mathcal{G}^{-1}(-\gamma)$ is a three-dimensional hyperboloid that is homeomorphic to a solid torus, and the reduced space associated to Hamiltonian $\mathcal{G}$ is the two-dimensional hyperboloid of revolution

$$
\mathcal{B}(\gamma)=\left\{\left(b_{1}, b_{2}, b_{3}\right) \in \mathbb{R}^{3}: b_{1} b_{2}-b_{3}^{2}=\gamma^{2}\right\} .
$$

In the invariants (25) the Hamiltonian (24) assumes the form

$$
\mathcal{H}_{\varepsilon}=b_{4}+\varepsilon^{3}\left(\frac{b_{2}}{2}-\frac{1}{\sqrt{b_{1}}}\right)+O\left(\varepsilon^{5}\right)
$$


Now, to obtain the Hamiltonian on the reduced space $\mathcal{B}(\gamma)$ we drop the constant $\gamma$ as well as terms $O\left(\varepsilon^{5}\right)$ and divide by $\varepsilon^{2}$, arriving at

$$
\overline{\mathcal{H}}=\frac{b_{2}}{2}-\frac{1}{\sqrt{b_{1}}} .
$$

Thus, the equations of motion of $\overline{\mathcal{H}}$ obtained from $\dot{b}_{j}=\sum_{k=1}^{3}\left\{b_{j}, b_{k}\right\} \partial \overline{\mathcal{H}} / \partial b_{k}$ and (26) are

$$
\dot{b}_{1}=2 b_{3}, \quad \dot{b}_{2}=-2 b_{3}\left(b_{1}\right)^{-3 / 2}, \quad \dot{b}_{3}=-\left(b_{1}\right)^{-1 / 2}+b_{2} .
$$

Proposition 3.1. On the reduced space $\mathcal{B}(\gamma)$ with $\gamma \neq 0$, system (24) has a unique critical point given by $\left(\gamma^{4}, \gamma^{-2}, 0\right)$.

Proof. It follows directly from the solution of system (30), taking into account the restrictions of (25) and $b_{1} b_{2}-b_{3}^{2}=\gamma^{2}$.

3.2. Symplectic coordinates on the reduced space $\mathcal{B}(\gamma)$. Here, we define symplectic coordinates in the neighborhoods of the critical points of Proposition 3.1 through the transformations $S^{ \pm}: \Omega^{ \pm} \rightarrow \mathbb{R}^{2}$ given by

$$
Q=\sqrt{2(L \pm \gamma)} \sin \ell, \quad P=\sqrt{2(L \pm \gamma)} \cos \ell,
$$

where $\Omega^{ \pm}=\{(\gamma, L): \pm \gamma \leq L\}$. The sign "+" applies for $\gamma<0$ (prograde motions), whereas the sign "-" is used when $\gamma>0$ (retrograde motions). The maps $\psi_{j}: V_{j} \rightarrow \mathbb{R}^{2}, \psi_{j}(\mathbf{b})=(Q, P)$ defined by equations (31), where $V_{j}=\left\{\mathbf{b}=\left(b_{1}, b_{2}, b_{3}, b_{4}\right) \in \mathcal{B}(\gamma): b_{4}=(-1)^{j} \gamma\right\}$ for $j=1,2$, are local charts for the reduced space $\mathcal{B}(\gamma)$.

Hamiltonian (29) in the coordinates $(Q, P)$ introduced through the atlas $\mathcal{A}=\left\{\psi_{1}, \psi_{2}\right\}$ is

$$
\overline{\mathcal{H}}=\frac{2}{\left(P^{2}+Q^{2} \mp 2 \gamma\right)^{2}},
$$

and the critical point given in Proposition 3.1 corresponds to the origin in $\mathbb{R}^{2}$.

3.3. Periodic solutions and KAM tori. In what follows we denote by

$$
p(t, \varepsilon)=\left(x_{1}(t, \varepsilon), x_{2}(t, \varepsilon), y_{1}(t, \varepsilon), y_{2}(t, \varepsilon)\right)
$$

a solution of system $(24)$ and by $p^{*} \in \mathcal{N}_{0}(\gamma)$ the periodic solution associated to the critical point $\bar{p} \in \mathcal{B}(\gamma)$.

Theorem 3.1. The charged restricted circular three-body problem has two families of near-circular periodic solutions linearly stable with characteristic multipliers $1,1,1 \pm \varepsilon^{3} \gamma^{-3} T i+O\left(\varepsilon^{6}\right)$. The radii and periods of these solutions are very large, $\|x\| \approx \varepsilon^{-2} \gamma^{2}$ and $T(\varepsilon) \approx 2 \pi \varepsilon^{-1}$.

Proof. First, we note that the Hamiltonian $\overline{\mathcal{H}}$ has Taylor expansion

$$
\overline{\mathcal{H}}=-\frac{1}{2 \gamma^{2}} \mp \frac{1}{2 \gamma^{3}}\left(Q^{2}+P^{2}\right)-\frac{3}{8 \gamma^{4}}\left(Q^{2}+P^{2}\right)^{2}+O(6) .
$$

Thence, it is clear that the Hessian matrix $D^{2} \overline{\mathcal{H}}$ at the origin is non-degenerate. Thus, by virtue of Reeb's Theorem 6.2, the proof of the first part of our theorem follows. Clearly, the matrix $A=\mathbb{J} D^{2} \overline{\mathcal{H}}$ is strongly stable which implies that the periodic solutions are linearly stable.

Theorem 3.2. The near-circular periodic solutions of the charged comet problem are enclosed by $K A M$ 2-tori for small enough $\varepsilon$. In addition, the measure of the set of tori disappearing under the perturbation is exponentially small of order $O(\exp (-$ const $/ \varepsilon))$. 
Proof. After introducing action-angle coordinates $(I, \varphi)$ through $Q=\sqrt{2 I} \sin \varphi, P=\sqrt{2 I} \cos \varphi$, and taking in account that $\gamma=-G$, Hamiltonian (24) assumes the form

$$
\mathcal{H}_{\varepsilon}=h_{0}(G)+\varepsilon^{3} h_{1}(G, I)+O\left(\varepsilon^{6}\right),
$$

where

$$
h_{0}(G)=-G, \quad h_{1}(G, I)=-\frac{1}{2(I \pm G)^{2}} .
$$

Now, since $\frac{\partial h_{0}}{\partial G}=-1, \frac{\partial h_{1}}{\partial I}=(I \pm G)^{-3}$ and $\frac{\partial^{2} h_{1}}{\partial I^{2}}=-3(I \pm G)^{-4}$ are nonzero, the perturbation removes the degeneracy. Thus, by virtue of Neishtadt's version of Arnold's Theorem for Hamiltonians with proper degeneracy (Theorem 6.20 in [3]), the result is proved.

Observation 3.1. Instead of Arnold-Neishtadt's Theorem [3], we can apply Han-Li-Yi's Theorem [14] on the Hamiltonian (33). Using the notation in Theorem 6.4 and taking $n=2, a=1, m_{1}=3$, $n_{0}=1, n_{1}=2, I^{n_{0}}=\bar{I}^{n_{0}}=L, I^{n_{1}}=(G, I)$, and $\bar{I}^{n_{1}}=I$, the frequency vector associated to (33) becomes

$$
\Omega(G, I)=\left(-1,-(I \pm G)^{-3}\right)
$$

and the $2 \times 2$ matrix with columns $\Omega, \partial \Omega / \partial G, \partial \Omega / \partial I$ given by

$$
M_{\Omega}=\left[\begin{array}{ccc}
-1 & 0 & 0 \\
-(I \pm G)^{-3} & \pm 3(I \pm G)^{-4} & 3(I \pm G)^{-4}
\end{array}\right]
$$

has rank two. In consequence, Han-Li-Yi's Theorem guarantees the existence of KAM tori of dimension 2 enclosing the near-circular periodic solutions. According to Theorem 6.4 we have $b=3$ and $s=1$, therefore, the excluded measure for the existence of quasi-periodic invariant tori is of order $O\left(\varepsilon^{3}\right)$.

\section{Poincaré charged problem}

The charged restricted circular three-body problem given in (1) has a parameter $\mu$, the mass ratio parameter for which when $\mu=0$, the problem is just the Kepler problem in rotating coordinates. Consider $\mu$ as a small parameter; so, Hamiltonian (1) assumes the form

$$
\mathcal{H}_{\mu}=\frac{1}{2}\left(y_{1}^{2}+y_{2}^{2}\right)-\frac{\beta_{1}^{3}}{\sqrt{x_{1}^{2}+x_{2}^{2}}}-\left(x_{1} y_{2}-x_{2} y_{1}\right)+O(\mu) .
$$

4.1. Continuation of periodic solutions. For $\mu=0$ Hamiltonian (34) is the Kepler problem in rotating coordinates. Introducing polar coordinates this Hamiltonian assumes the form

$$
\mathcal{H}_{0}=\frac{1}{2}\left(R^{2}+\frac{\Theta^{2}}{r^{2}}\right)-\Theta-\frac{\beta_{1}^{3}}{r}
$$

with associated vector field

$$
\begin{array}{ll}
\dot{r}=R, & \dot{R}=\frac{\Theta^{2}}{r^{3}}-\frac{\beta_{1}^{3}}{r^{2}}, \\
\dot{\theta}=\frac{\Theta}{r^{2}}-1, & \dot{\Theta}=0 .
\end{array}
$$

Thence, it is clear that $\Theta$ is a integral for Hamiltonian (35). Next, fixing $\Theta=\gamma$ with $\gamma \neq 0$ we have the following result.

Theorem 4.1. If $\gamma \neq \beta_{1}^{2}$ and $1 /\left(1-\gamma^{3} \beta_{1}^{-6}\right) \notin \mathbb{Z}$, then the circular solutions of the Kepler problem in rotating coordinates with angular momentum $\gamma$ can be continued into the restricted problem for small values of $\mu$. These solutions are linearly stable with periods near to $T=\left|2 \pi \gamma^{3} /\left(\beta_{1}^{6}-\gamma^{3}\right)\right|$. 
Proof. Considering $\Theta=\gamma$ we have that $\mathcal{O}_{\gamma}=\left(\gamma^{2} / \beta_{1}^{3}, 0\right)$ is an equilibrium point for the system

$$
\dot{r}=R, \quad \dot{R}=\frac{\Theta^{2}}{r^{3}}-\frac{\beta_{1}^{3}}{r^{2}},
$$

Now, linearizing this system around of $\mathcal{O}_{\gamma}$ we obtain

$$
\dot{r}=R, \quad \dot{R}=-\left(\beta_{1}^{2} \gamma^{-1}\right)^{6} r
$$

or equivalently the oscillator equation

$$
\ddot{r}+\left(\beta_{1}^{2} \gamma^{-1}\right)^{6} r=0 .
$$

Solving this equation we obtain solutions of the form $r(t)=e^{ \pm i\left(\beta_{1}^{2} \gamma^{-1}\right)^{3} t}$. Hence, the nontrivial characteristic multipliers are $e^{ \pm 2 \pi i /\left(1-\gamma^{3} \beta_{1}^{-6}\right)}$ which are not 1 , provided $1 /\left(1-\gamma^{3} \beta_{1}^{-6}\right)$ is not an integer. Thus, the circular orbits can be continued for small values of $\mu$. Since $r=\gamma^{2} / \beta_{1}^{3}, R=0$ corresponds to a circular solution with period $T=\left|2 \pi \gamma^{3} /\left(\beta_{1}^{6}-\gamma^{3}\right)\right|$ for system (36), the continued circular solutions have periods near to $T$.

4.2. Symmetric periodic solutions. In Delaunay coordinates an orthogonal crossing of the line of syzygy at a time $t_{0}$ is

$$
\ell\left(t_{0}\right)=\pi n_{1}, \quad g\left(t_{0}\right)=\pi n_{2}, \quad n_{1}, n_{2} \in \mathbb{Z} .
$$

In these coordinates Hamiltonian (34) becomes

$$
\mathcal{H}=-\frac{1}{2 L^{2}}-G+O(\mu)
$$

and the associated equations of motion are

$$
\begin{array}{ll}
\dot{\ell}=1 / L^{3}+O(\mu), & \dot{L}=O(\mu), \\
\dot{g}=-1+O(\mu), & \dot{G}=O(\mu) .
\end{array}
$$

We designate by $\varphi\left(t, \mathbf{Y}_{0}, \mu\right)=(\ell(t, \mu), g(t, \mu), L(t, \mu), G(t, \mu))$ a solution of system (1) in Delaunay coordinates with initial condition $\mathbf{Y}_{0}=\left(\pi n_{1}, \pi n_{2}, L_{0}, G_{0}\right)$. Besides, with respect to this initial condition we introduce the notation $\mathbf{Y}_{\mu}=\left(\pi n_{1}, \pi n_{2}, L_{0}+O(\mu), G_{0}+O(\mu)\right)$.

Theorem 4.2. Let $m, n$ be relatively prime integers and $T=2 \pi m$. Then the elliptic $T$-periodic solution of the Poincaré charged problem in rotating coordinates with initial condition $\mathbf{Y}_{0}=\left(\pi n_{1}, \pi n_{2}\right.$, $\left.L_{0}, G_{0}\right)$ such that $L_{0}^{3}=m / n$ and $G_{0} \in \mathbb{R}$ can be continued for $\mu$ small. More precisely, for $\mu$ sufficiently small there is an initial condition $\mathbf{Y}_{\mu}$ such that $\varphi\left(t, \mathbf{Y}_{\mu}, \mu\right)$ is a $\mathcal{S}_{1}$-symmetric solution with period $T(\mu)$ near to $T$.

Proof. Let $\varphi(t, \mathbf{Y}, \mu)$ be an elliptic solution of system (39) with initial condition $\mathbf{Y}=\left(\pi n_{1}, \pi n_{2}, L\right.$, $G)$ on the line of syzygy in a neighborhood of $\mathbf{Y}_{0}$. We obtain

$$
\ell(t, \mathbf{Y}, \mu)=\pi n_{1}+t / L^{3}+O(\mu), \quad g(t, \mathbf{Y}, \mu)=\pi n_{2}-t+O(\mu) .
$$

Thus,

$$
\ell\left(T / 2, \mathbf{Y}_{0}, 0\right)=\pi\left(n_{1}+n\right), \quad g\left(T / 2, \mathbf{Y}_{0}, 0\right)=\pi\left(n_{1}-m\right),
$$

which implies that when $\mu=0$ the solution has another orthogonal crossing at time $T / 2=\pi m$ and then it is $\mathcal{S}_{1}$-symmetric. On the other hand, since the determinant

$$
\left.\frac{\partial(\ell, g)}{\partial(t, L)}\right|_{\left(t=T / 2, L=L_{0}, \mu=0\right)}=\left|\begin{array}{ll}
\partial \ell / \partial t & \partial \ell / \partial L \\
\partial g / \partial t & \partial g / \partial L
\end{array}\right|=\left|\begin{array}{cc}
n / m & -3 \pi\left(n^{4} / m\right)^{1 / 3} \\
-1 & 0
\end{array}\right|=-3 \pi\left(\frac{n^{4}}{m}\right)^{1 / 3},
$$

is non-zero, by the implicit function theorem the result follows. 
Theorem 4.3. The families of symmetric periodic solutions of the Poincaré charged problem are enclosed by invariant KAM 2-tori for small enough $\mu$. The measure of the set of tori that do not persist under the perturbation is of order $O(\sqrt{\varepsilon})$.

Proof. We consider a symmetric periodic solution $\varphi\left(t, \mathbf{Y}_{\mu}, \mu\right)$ with initial condition

$$
\mathbf{Y}_{\mu}=\left(\pi n_{1}, \pi n_{2}, L_{0}+O(\mu), G_{0}+O(\mu)\right)
$$

as in Theorem 4.2. Denoting by $\mathcal{H}_{0}$ the unperturbed Hamiltonian associated to (38) we arrive at the determinant

$$
\operatorname{det}\left[\begin{array}{ccc}
\frac{\partial^{2} \mathcal{H}_{0}}{\partial L^{2}} & \frac{\partial^{2} \mathcal{H}_{0}}{\partial L \partial G} & \frac{\partial \mathcal{H}_{0}}{\partial L} \\
\frac{\partial^{2} \mathcal{H}_{0}}{\partial G \partial L} & \frac{\partial^{2} \mathcal{H}_{0}}{\partial G^{2}} & \frac{\partial \mathcal{H}_{0}}{\partial G} \\
\frac{\partial \mathcal{H}_{0}}{\partial L} & \frac{\partial \mathcal{H}_{0}}{\partial G} & 0
\end{array}\right]=\left|\begin{array}{ccc}
-3 L^{-4} & 0 & L^{-3} \\
0 & 0 & -1 \\
L^{-3} & -1 & 0
\end{array}\right|=3 L^{-4}
$$

is nonzero. Thus, Hamiltonian (38) is isoenergetically non-degenerate. Therefore, by virtue of Kolmogorov's Theorem [3] the proof is concluded.

Now, we designate by $\psi\left(t, \mathbf{Y}_{0}, \mu\right)=\left(Q_{1}(t, \mu), Q_{2}(t, \mu), P_{1}(t, \mu), P_{2}(t, \mu)\right)$ a solution of system (1) in Poincaré-Delaunay coordinates with initial condition $\mathbf{Y}_{0}=\left(\pi n, 0, p_{1}, p_{2}\right)$. In these coordinates Hamiltonian (34) is written as

$$
\mathcal{H}=-\frac{1}{2 P_{1}^{2}}-P_{1}+\frac{1}{2}\left(Q_{2}^{2}+P_{2}^{2}\right)+O(\mu),
$$

and the equations of motions are

$$
\begin{array}{ll}
\dot{Q}_{1}=1 / P_{1}^{3}-1+O(\mu), & \dot{P}_{1}=O(\mu), \\
\dot{Q}_{2}=P_{2}+O(\mu), & \dot{P}_{2}=-Q_{2}+O(\mu) .
\end{array}
$$

Again, as in previous lines we denote $\mathbf{Y}_{\mu}=\left(\pi n, 0, p_{1}+O(\mu), p_{2}+O(\mu)\right)$.

Theorem 4.4. Let $m \in \mathbb{Z}, p_{1} \in \mathbb{R}^{+}$with $p_{1} \neq 1$, and $T / 2=\pi m p_{1}^{3} /\left(1-p_{1}^{3}\right) \notin \pi \mathbb{Z}$. Then the circular T-periodic solution of the Kepler problem in rotating coordinates with initial condition $\mathbf{Y}_{0}=\left(\pi n, 0, p_{1}, 0\right)$ such that $p_{2} \in \mathbb{R}$ can be continued into the restricted problem for $\mu$ small. More precisely, for $\mu$ sufficiently small there is an initial condition $\mathbf{Y}_{\mu}$ such that $\psi\left(t, \mathbf{Y}_{\mu}, \mu\right)$ is a $\mathcal{S}_{1}$-symmetric solution with fixed period $T$.

Proof. Let $\psi\left(t, \mathbf{Y}_{\mu}, \mu\right)$ be a circular solution of system (42) with initial condition $\mathbf{Y}=\left(\pi n, 0, P_{1}, P_{2}\right)$ in a neighborhood of $\mathbf{Y}_{0}$. Thus, the solution of system (42) is

$$
Q_{1}(t, \mathbf{Y}, \mu)=\pi n+\left(1 / P_{1}^{3}-1\right) t+O(\mu), \quad Q_{2}(t, \mathbf{Y}, \mu)=P_{2} \sin t+O(\mu),
$$

and thence,

$$
Q_{1}\left(T / 2, \mathbf{Y}_{0}, 0\right)=\pi(n+m), \quad Q_{2}\left(T / 2, \mathbf{Y}_{0}, 0\right)=0
$$

which implies that this solution has another orthogonal crossing at time $T / 2=\pi m p_{1}^{3} /\left(1-p_{1}^{3}\right)$ for $\mu=0$ and then it is $\mathcal{S}_{1}$-symmetric. Now, since $T / 2 \notin \pi \mathbb{Z}$ the determinant

$$
\left.\frac{\partial\left(Q_{1}, Q_{2}\right)}{\partial\left(P_{1}, P_{2}\right)}\right|_{\left(t=T / 2, P_{1}=p_{1}, P_{2}=0, \mu=0\right)}=\left|\begin{array}{cc}
-3 p_{1}^{-4} & 0 \\
0 & \sin (T / 2)
\end{array}\right|=-3 p_{1}^{-4} \sin (T / 2)
$$

is nonzero. Therefore, by the implicit function theorem the proof of the theorem follows. 


\section{Hamiltonian-Hopf Bifurcations}

Now we study the bifurcations of the triangular equilibrium points $L_{4}^{i s o}$ and $L_{5}^{i s o}$ found in [29]. More precisely, we will prove that in the Hamiltonian associated to each triangular equilibrium point a Hamiltonian-Hopf bifurcation occurs.

Here, we will treat bifurcations of the triangular equilibrium points in the isosceles case, or equivalently, when $\beta_{1}=\beta_{2}$ with $\beta_{1}>1 / 2$. Setting $\beta_{1}=\beta_{2}=\beta$, the isosceles equilibrium points are parametrized by

$$
\begin{aligned}
& L_{4}^{i s o}=\left(\frac{1}{2}-\mu, \frac{1}{2} \sqrt{4 \beta^{2}-1},-\frac{1}{2} \sqrt{4 \beta^{2}-1}, \frac{1}{2}-\mu\right), \\
& L_{5}^{\text {iso }}=\left(\frac{1}{2}-\mu,-\frac{1}{2} \sqrt{4 \beta^{2}-1}, \frac{1}{2} \sqrt{4 \beta^{2}-1}, \frac{1}{2}-\mu\right) .
\end{aligned}
$$

At this point it is convenient to define the function $F: M=(0,1 / 2] \times(1 / 2, \infty) \rightarrow \mathbb{R}$ by

$$
F(\mu, \beta)=9 \mu(1-\mu) \frac{4 \beta^{2}-1}{\beta^{4}},
$$

and the parametric sets:

$$
\left.\mathcal{S}^{-}=F^{-1}((0,1)), \quad \mathcal{C}=F^{-1}(\{1\}), \quad \mathcal{S}^{+}=F^{-1}(1, \infty)\right),
$$

as well as the parameters

$$
\omega_{1}=\sqrt{\frac{1}{2}(1+\sqrt{1-F(\mu, \beta)})} \quad \text { and } \quad \omega_{2}=\sqrt{\frac{1}{2}(1-\sqrt{1-F(\mu, \beta)})} .
$$

According to Section 3 of paper [29], the linear part of Hamiltonian system at $L_{4}^{\text {iso }}$ and $L_{5}^{\text {iso }}$ has eigenvalues given by $\pm i \omega_{1}, \pm i \omega_{2}$, which are pure imaginary for $(\mu, \beta) \in \mathcal{S}^{-}$, complex with nonzero real part for $(\mu, \beta) \in \mathcal{S}^{+}$, and $\pm i \sqrt{2} / 2$ (with multiplicity two) for $(\mu, \beta) \in \mathcal{C}$. This is the typical scenario of a Hamiltonian-Hopf bifurcation occurring at $(\mu, \beta) \in \mathcal{C}$.

Theorem 5.1. The parametric curve $\mathcal{C}$ (1:-1 resonance) corresponds to supercritical HamiltonianHopf bifurcations of $L_{4}^{\text {iso }}$ and $L_{5}^{\text {iso }}$. More precisely, there are two families of elliptic periodic solutions with period near to $2 \pi$ emanating from $L_{4}^{\text {iso }}$ and $L_{5}^{\text {iso }}$. One family exists for $\mathcal{H}>0$ and the other for $\mathcal{H}<0$.

Proof. For values $(\mu, \beta) \in \mathcal{C}$ the matrix associated to the quadratic part of $\mathcal{H}$ is non-diagonalizable and the frequencies $\omega_{1}=\omega_{2}=\sqrt{2} / 2$ are in 1:-1 resonance. Also, for $(\mu, \beta) \in \mathcal{C}$, we have that

$$
0<\mu \leq \frac{1}{2} \quad \text { and } \quad \sqrt{\frac{3}{2}(3-2 \sqrt{2})} \leq \beta \leq \sqrt{\frac{3}{2}(3+2 \sqrt{2})} .
$$

Linearizing Hamiltonian (1) around $L_{4}^{\text {iso }}$ and $L_{5}^{\text {iso }}$, adding higher-order terms, bringing the quadratic part to canonical form applying the symplectic linear changes of [20] S. 11.5, and transforming the nonlinear terms accordingly, applying also the nonlinear normalization procedure, the corresponding normal form becomes

$$
\begin{aligned}
\mathcal{H}= & \frac{1}{2}\left(x_{1}^{2}+x_{2}^{2}\right)+\frac{\sqrt{2}}{2}\left(x_{1} y_{2}-x_{2} y_{1}\right)+a(\beta)\left(y_{1}^{2}+y_{2}^{2}\right)^{2}+b(\beta)\left(x_{1} y_{2}-x_{2} y_{1}\right)\left(y_{1}^{2}+y_{2}^{2}\right) \\
& +c(\beta)\left(x_{1} y_{2}-x_{2} y_{1}\right)^{2}+\ldots,
\end{aligned}
$$

where

$$
\begin{gathered}
a(\beta)=\frac{36 \beta^{6}+78 \beta^{4}-70 \beta^{2}+15}{9 \beta^{6}\left(4 \beta^{2}-1\right)}, \quad b(\beta)=-\frac{\sqrt{2}\left(576 \beta^{6}+1434 \beta^{4}-1235 \beta^{2}+255\right)}{216 \beta^{6}\left(4 \beta^{2}-1\right)}, \\
c(\beta)=\frac{504 \beta^{6}-2334 \beta^{4}+1385 \beta^{2}-210}{324\left(4 \beta^{2}-1\right)} \\
12
\end{gathered}
$$


Since the coefficients of $x_{1} y_{2}-x_{2} y_{1}$ and $\left(y_{1}^{2}+y_{2}^{2}\right)^{2}$ are non-null for $(\mu, \beta) \in \mathcal{C}$, by virtue of Theorem 6.5 we conclude that a Hamiltonian-Hopf bifurcation takes place. The sign of the coefficient of $\left(y_{1}^{2}+y_{2}^{2}\right)^{2}$ determines the type of bifurcation. In our case, as the coefficient $a(\beta)$ is positive because $(\mu, \beta) \in \mathcal{C}$, the bifurcation is supercritical.

\section{Appendix}

Consider the linear Hamiltonian system

$$
\dot{z}=A z=\mathbb{J} \nabla \mathcal{H}(z), \quad \mathcal{H}=\frac{1}{2} z^{T} S z,
$$

where $S$ is a symmetric constant matrix and $A=\mathbb{J} S$ is a Hamiltonian matrix.

Definition 6.1 (Strong stability). System (45) (or the matrix A) is strongly stable (or parametrically stable) if it and all sufficiently small linear constant Hamiltonian perturbations of it are stable. If system (45) is stable but not strongly stable, we say that it is weakly stable.

Let $\pm \alpha_{1} i, \pm \alpha_{2} i, \ldots, \pm \alpha_{s} i$ be the eigenvalues of the matrix $A$, and let $V_{j}, j=1, \ldots, s$, be the maximal real linear subspace where $A$ has eigenvalues $\pm \alpha_{j} i$. So $V_{j}$ is an $A$-invariant symplectic subspace, $A$ restricted to $V_{j}$ has eigenvalues $\pm \alpha_{j} i$, and $\mathbb{R}^{2 n}=V_{1} \oplus V_{2} \oplus \ldots \oplus V_{s}$. Let $\mathcal{H}_{j}$ be the restriction of $\mathcal{H}$ to $V_{j}$.

Theorem 6.1 (Krein-Gel'fand). System (45) is strongly stable if and only if

- all the eigenvalues of $A$ are purely imaginary,

- $A$ is nonsingular,

- A is diagonalizable over the complex numbers, and

- Hamiltonian $\mathcal{H}_{j}$ is positive or negative definite for each $j$.

See the proof in [27] or in [20].

Let $(M, \Omega)$ be a symplectic manifold of dimension $2 n, \mathcal{H}_{0}: M \rightarrow \mathbb{R}$ a smooth Hamiltonian which defines a Hamiltonian vector field $Y_{0}=\left(d H_{0}\right)^{\#}$ with symplectic flow $\varphi_{0}^{t}$. Let $I \subset \mathbb{R}$ be an interval such that each $h \in I$ is a regular value of $\mathcal{H}_{0}$ and $\mathcal{N}_{0}(h)=\mathcal{H}_{0}^{-1}(h)$ is a compact connected circle bundle over a base space $\mathcal{B}(h)$ with projection $\pi: \mathcal{N}_{0}(h) \rightarrow \mathcal{B}(h)$. So, this is the setting of regular reduction theory. Assume that all the solutions of $Y_{0}$ in $\mathcal{N}_{0}(h)$ are periodic and have periods smoothly depending only on the value of the Hamiltonian; i.e., the period is a smooth function $T=T(h)$.

Let $\varepsilon$ be a small parameter, $\mathcal{H}_{1}: M \rightarrow \mathbb{R}$ be smooth, $\mathcal{H}_{\varepsilon}=\mathcal{H}_{0}+\varepsilon \mathcal{H}_{1}, Y_{\varepsilon}=Y_{0}+\varepsilon Y_{1}=d \mathcal{H}_{\varepsilon}^{\#}$, $\mathcal{N}_{\varepsilon}(h)=\mathcal{H}_{\varepsilon}^{-1}(h), \pi: \mathcal{N}_{\varepsilon}(h) \rightarrow \mathcal{B}(h)$ the projection, and $\phi_{\varepsilon}^{t}$ be the flow defined by $Y_{\varepsilon}$.

Let the average of $\mathcal{H}_{1}$ be

$$
\overline{\mathcal{H}}=\frac{1}{T} \int_{0}^{T} \mathcal{H}_{1}\left(\phi_{0}^{t}\right) d t .
$$

The next result provides sufficient conditions for characterising the existence of periodic solutions of the Hamiltonian system associated to $\mathcal{H}_{\varepsilon}$. For more information on this subject the reader is addressed to [26], [28] and [21].

Theorem 6.2 (Reeb). If $\overline{\mathcal{H}}$ has a non-degenerate critical point at $\pi(p)=\bar{p} \in \mathcal{B}(h)$ with $p \in \mathcal{N}_{0}(h)$, then there are smooth functions $p(\varepsilon)$ and $T(\varepsilon)$ for $\varepsilon$ small with $p(0)=p, T(0)=T$, and $p(\varepsilon) \in \mathcal{N}_{\varepsilon}$, and the solution of $Y_{\varepsilon}$ through $p(\varepsilon)$ is $T(\varepsilon)$-periodic. In addition, if the characteristic exponents of 
the critical point $\bar{p}$ (that is, the eigenvalues of the matrix $A=\mathbb{J} D^{2} \overline{\mathcal{H}}(\bar{p})$ ) are $\lambda_{1}, \lambda_{2}, \ldots, \lambda_{2 n-2}$, then the characteristic multipliers of the periodic solution through $p(\varepsilon)$ are

$$
1,1,1+\varepsilon \lambda_{1} T+O\left(\varepsilon^{2}\right), 1+\varepsilon \lambda_{2} T+O\left(\varepsilon^{2}\right), \ldots, 1+\varepsilon \lambda_{2 n-2} T+O\left(\varepsilon^{2}\right) .
$$

Theorem 6.3. Let $p$ and $\bar{p}$ as in the previous theorem. If one or more of the characteristic exponents $\lambda_{j}$ is real or has nonzero real part, then the periodic solution through $p(\varepsilon)$ is unstable. If the matrix $A$ is strongly stable, then the periodic solution through $p(\varepsilon)$ is elliptic, i.e., linearly stable.

The proofs of Theorems 6.2 and 6.3 appear in [28].

Consider a Hamiltonian system of the form

$$
\mathcal{H}_{\varepsilon}(I, \varphi, \varepsilon)=h_{0}\left(I^{n_{0}}\right)+\varepsilon^{m_{1}} h_{1}\left(I^{n_{1}}\right)+\ldots+\varepsilon^{m_{a}} h_{a}\left(I^{n_{a}}\right)+\varepsilon^{m_{a}+1} p(I, \varphi, \varepsilon),
$$

where $(I, \varphi) \in \mathbb{R}^{n} \times \mathbb{T}^{n}$ are action-angle coordinates with the standard symplectic structure $d I \wedge d \varphi$, and $\varepsilon>0$ is a sufficiently smal parameter. Hamiltonian $\mathcal{H}_{\varepsilon}$ is real analytic, and the parameters $a, m, n_{i}(i=0,1, \ldots, a)$ and $m_{j}(j=1,2, \ldots, a)$ are positive integers satisfying $n_{0} \leq n_{1} \leq \ldots \leq$ $n_{a}=n, m_{1} \leq m_{2} \leq \ldots \leq m_{a}=m, I^{n_{i}}=\left(I_{1}, \ldots, I_{n_{i}}\right)$, for $i=1,2, \ldots, a$, and $p$ depends on $\varepsilon$ smoothly.

Hamiltonian $\mathcal{H}_{\varepsilon}(I, \varphi, \varepsilon)$ is taken in a bounded closed region $Z \times \mathbb{T}^{n} \subset \mathbb{R}^{n} \times \mathbb{T}^{n}$. For each $\varepsilon$ the integrable part of $\mathcal{H}_{\varepsilon}$,

$$
X_{\varepsilon}(I)=h_{0}\left(I^{n_{0}}\right)+\varepsilon^{m_{1}} h_{1}\left(I^{n_{1}}\right)+\ldots+\varepsilon^{m_{a}} h_{a}\left(I^{n_{a}}\right),
$$

admits a family of invariant $n$-tori $T_{\zeta}^{\varepsilon}=\{\zeta\} \times \mathbb{T}^{n}$, with linear flows $\left\{x_{0}+\omega^{\varepsilon}(\zeta) t\right\}$, where, for each $\zeta \in Z, \omega^{\varepsilon}(\zeta)=\nabla X_{\varepsilon}(\zeta)$ is the frequency vector of the $n$-torus $T_{\zeta}^{\varepsilon}$ and $\nabla$ is the gradient operator. When $\omega^{\varepsilon}(\zeta)$ is nonresonant, the $n$-torus $T_{\zeta}^{\varepsilon}$ becomes quasi-periodic with slow and fast frequencies of different scales. We refer to the integrable part $X_{\varepsilon}$ and its associated tori $\left\{T_{\zeta}^{\varepsilon}\right\}$ as the intermediate Hamiltonian and intermediate tori, respectively.

Let $\bar{I}^{n_{i}}=\left(I_{n_{i-1}+1}, \ldots, I_{n_{i}}\right), i=0,1, \ldots, a$ (where $n_{-1}=0$, hence $\left.\bar{I}^{n_{0}}=I^{n_{0}}\right)$, and define

$$
\Omega=\left(\nabla_{\bar{I}^{n_{0}}} h_{0}\left(I^{n_{0}}\right), \ldots, \nabla_{\bar{I}^{n_{a}}} h_{n_{a}}\left(I^{n_{a}}\right)\right),
$$

such that, for each $i=0,1, \ldots, a, \nabla_{\bar{I}^{n_{i}}}$ denotes the gradient with respect to $\bar{I}^{n_{i}}$.

We assume the following high-order degeneracy-removing condition of Bruno-Rüssman type (so named by Han, Li and Yi), giving credit to Bruno and Rüssman, who provided weak conditions on the frequencies guaranteeing the persistence of invariant tori, the so-called $(A)$ condition: there is a positive integer $s$ such that

$$
\operatorname{Rank}\left\{\partial^{\alpha} \Omega(I): 0 \leq|\alpha| \leq s\right\}=n, \quad \forall I \in Z .
$$

For the usual case of a nearly integrable Hamiltonian system of the type

$$
\mathcal{H}_{\varepsilon}(I, \varphi, \varepsilon)=X(I)+\varepsilon p(I, \varphi, \varepsilon), \quad(I, \varphi) \in Z \times \mathbb{T}^{n} \subset \mathbb{R}^{n} \times \mathbb{T}^{n} .
$$

Condition $(A)$ given above generalises the classical Kolmogorov non-degenerate condition that $\partial \Omega(I)$ be nonsingular over $Z$, where $\Omega(I)=\nabla X(I)$; Bruno's non-degenerate condition that $\operatorname{Rank}\{\Omega(I), \partial \Omega\}=n, \forall I \in Z$; and the weakest non-degenerate condition guaranteeing such persistence provided by Rüssman, that $\omega(Z)$ should not lie in any $(n-1)$-dimensional subspace. Rüssman condition is equivalent to condition $(A)$ for systems like (47). However, Bruno or Rüssman conditions do not apply to Hamiltonian (46), as it is too degenerate.

The following theorem gives the right setting in which one can ensure the persistence of KAM tori for Hamiltonian like (46). 
Theorem 6.4 (Han, Li and Yi). Assume the condition $(A)$, and let $\delta$ with $0<\delta<1 / 5$ be given. Then there exists an $\varepsilon_{0}>0$ and a family of Cantor sets $Z_{\varepsilon} \subset Z, 0<\varepsilon<\varepsilon_{0}$, with $\left|Z \backslash Z_{\varepsilon}\right|=$ $O\left(\varepsilon^{\delta / s}\right)$, such that each $\zeta \in Z_{\varepsilon}$ corresponds to a real analytic, invariant, quasi-periodic $n$-torus $\bar{T}_{\zeta}^{\varepsilon}$ of Hamiltonian (46), which is slightly deformed from the intermediate $n$-torus $T_{\zeta}^{\varepsilon}$. Moreover, the family $\left\{\bar{T}_{\zeta}^{\varepsilon}: \zeta \in Z_{\varepsilon}, 0<\varepsilon<\varepsilon_{0}\right\}$ varies Whitney smoothly.

See the proof in [14].

Consider the Hamiltonian in 1:-1 resonance

$$
\mathcal{H}=\frac{\omega}{2}\left(x_{1} y_{2}-x_{2} y_{1}\right)+\delta\left(x_{1}^{2}+x_{2}^{2}\right)+\Gamma\left(y_{1}^{2}+y_{2}^{2}\right)^{2}+\ldots,
$$

where $\omega \neq 0$ and $\delta= \pm 1$.

Theorem 6.5. Given the parametric family defined by (48) there are periodic solutions emanating from the origin when $\delta \Gamma>0$. One family exists for $\mathcal{H}>0$ and one for $\mathcal{H}<0$. There are no nearby $2 \pi$-periodic solutions when $\delta \Gamma<0$.

See the proof in $[22]$.

\section{REFERENCES}

[1] F. Alfaro, E. PÉrez-Chavela, Relative equilibria in the charged n-body problem, Can. Appl. Math. Q., 10, (2002), 1, 1-13.

[2] F. Alfaro, E. PÉrez-Chavela, Linear stability of relative equilibria in the charged three body problem, J. Differential Equations, 245, (2008), 1923-1944.

[3] V. Arnold, V. Kozlov, A. Neishtadt, Mathematical Aspects of Classical and Celestial Mechanics Encyclopaedia of Mathematical Sciences, Vol 3. Dynamical Systems III - Third Edition. Springer-Verlag Berlin Heidelberg (2006).

[4] P. Atela, The charged isosceles 3-body problem, Contemporary Mathematics, 81 (1988), 43-58.

[5] A. Bengochea, C. Vidal, On a planar circular restricted charged three-body problem, Astrophysics and Space Science, 358: 9 (2015).

[6] J. Casasayas, A. Nunes, A restricted charged four-body problem, Celestial Mech. Dynam. Astronom., 47 (1990), 245-266.

[7] A. Castro, E. Lacomba, Non-hyperbolic equilibria in the charged collinear three-body problem, J. Dynam. Differential Equations, 28 (2012), 85-100.

[8] A. Castro, M. Falconi, Schubart solutions in the charged collinear three-body problem, J. Dynam. Differential Equations, 28 (2016), 519-532.

[9] R.C. Churchill, M. Kummer, D.L. Rod, On averaging, reduction, and symmetry in Hamiltonian systems, J. Differential Equations. 49 (1983), 359-414.

[10] R.H. Cushman, L.M. Bates, Global Aspects of Classical Integrable Systems, Birkhäuser Verlag, Basel, 1997.

[11] A. Deprit, Canonical transformations depending on a small parameter, Celestial Mech. Dynam. Astronom., 1 (1969), 12-30.

[12] A. DepRit, The elimination of the parallax in satellite theory, Celestial Mech. Dynam. Astronom., 24(2) (1981), 111-153. DOI: $10.1007 /$ BF01229192.

[13] D.D. Dyonisiou, G. Antonacopoulos, Relativistic dynamics for three charged particles, Celestial Mech. Dynam. Astronom., 23, 109-117, (1981).

[14] Y. Han, Y. LI, Y. YI, Invariant tori in Hamiltonian systems with high order proper degeneracy, Ann. Henri Poincaré, 10 (2010), 1419-1436.

[15] H. Hanssmann, J.C. van Der Meer, Local and Semi-Local Bifurcations in Hamiltonian Dynamical Systems: Results and Examples, Lectures Notes in Math 1893, Springer-Verlag, New York, 2007.

[16] J. Llibre, D. PASCA, C. VAlls, Qualitative study of a charged restricted three-body problem, J. Differential Equations 255, (2013), 326-338.

[17] J. Llibre, D.J Tonon, Symmetric periodic orbits for the collinear charged 3-body problem, J. Math. Phys. 58 (2017), 022702, 19 pp. 
[18] J. Mansilla, C. Vidal, Geometric interpretation for the spectral stability in the charged three-body problem, Celestial Mechanics and Dynamical Astronomy, 113 (2012), 205-213.

[19] J. Marsden, A. Weinstein, Reduction of symplectic manifolds with symmetry, Rep. Math. Phys. 5 (1974), 121-130.

[20] K.R. Meyer, D. Offin, Introduction to Hamiltonian Dynamical Systems and the N-Body Problem, Third Edition, Springer International Publishing AG, New York, 2017.

[21] K.R. Meyer, J.F. Palacián, P. Yanguas, Geometric averaging of Hamiltonian systems: periodic solutions, stability, and KAM tori, SIAM J. Appl. Dyn. Syst. 10 (2011), 817-856.

[22] K.R. Meyer, J.F. Palacián, P. Yanguas, The elusive Liapunov periodic solutions, Qual. Theory Dyn. Syst. 14, (2015), 381-401.

[23] K.R. Meyer, J.F. Palacián, P. Yanguas, Invariant tori in the Lunar problem, Publ. Mat. Extra (2014), 353-394.

[24] J. Moser, Regularization of Kepler's problem and the averaging method on a manifold, Comm. Pure Appl. Math., 23 (1970), 609-636.

[25] E. PÉrez-Chavela, D. SaAri, A. Susin, Z. Yan, Central configurations in the charged three body problem, Hamiltonian dynamics and celestial mechanics (Seattle, WA, 1995), 137-153, Contemp. Math., 198, Amer. Math. Soc., Providence, RI, 1996.

[26] G. ReEB, Sur certaines propriétés topologiques des trajectoires des systèmes dynamiques, Acad. Roy. Sci. Lett. et Beaux-Arts de Belgique. Cl. des Sci. Mém. in $8^{\circ}$, Ser. 2, 27 (1952), No. 9.

[27] V.A. Yakubovich, V.M. Starzhinskit, Linear Differential Equations with Periodic Coefficients, John Wiley \& Sons, New York, 1975.

[28] P. Yanguas, J.F. Palacián, K.R. Meyer, H.S. Dumas, Periodic solutions in Hamiltonian systems, averaging, and the Lunar problem, SIAM J. Appl. Dyn. Syst. 7 (2008), 311-340.

[29] C. VidAL, J. VidARTE, Stability of the equilibrium solutions in a charged restricted circular three-body problem, J. Differential Equations 260, (2016), 5128-5173.

(J.F. Palacián) Departamento de Ingeniería Matemática e Informática And Institute of Advanced Materials (INAMAT), Universidad Pública de Navarra, 31006 Pamplona, Spain

E-mail address: palacian@unavarra.es

(C. Vidal) Grupo de Investigación en Sistemas Dinámicos y Aplicaciones-GiSda, Departamento de Matemática, Facultad de Ciencias, Universidad del Bío-Bío, Concepción, Viti Región, Chile

E-mail address: clvidal@ubiobio.cl

(J. Vidarte) Departamento de Matemática, Facultad de Ciencias, Universidad del Bío-Bío, ConCEPCión, VIII REgión, Chile

E-mail address: jvidarte@ubiobio.cl

(P. Yanguas) Departamento de Ingeniería Matemática e Informática and Institute of Advanced Materials (INAMAT), Universidad Pública de Navarra, 31006 Pamplona, Spain

E-mail address: yanguas@unavarra.es 